\title{
Contour interaction as a function of retinal eccentricity
}

\author{
GEORGE WOLFORD and LARRY CHAMBERS \\ Dartmouth College, Hanover, New Hampshire
}

\begin{abstract}
Targets (squares with a gap on one side) were presented $0^{\circ}, 2^{\circ}$, or $5^{\circ}$ from the center of the fovea. The targets were surrounded by bar masks, and the spacing between targets and masks was varied parametrically. The resulting functions were used to estimate the extent of contour interaction at different target eccentricities. Our estimates for the extent of contour interaction were approximately $.1^{\circ}$ for targets at the center of the fovea, $.24^{\circ}$ for targets located $2^{\circ}$ from the center, and $.8^{\circ}$ for targets $5^{\circ}$ into the periphery.
\end{abstract}

Lateral masking refers to the finding that the probability of correctly identifying a target is reduced when that target is surrounded by other items. Lateral masking has been demonstrated by a number of investigators (e.g., Bouma, 1970; Estes \& Wolford, 1971; Mackworth, 1965). In this paper, we examine the effect of target-mask separation at different retinal eccentricities.

In a recent paper, we argued that lateral masking was a composite of at least three components. One component was sensory interaction at the level of contours or features (Flom, Weymouth, \& Kahneman, 1963). We will refer to this as contour interaction. A second component was the allocation of some attention or capacity to the masks that might otherwise be available for the target (Banks, Larson, \& Prinzmetal, 1979; Kahneman \& Henik, 1977). This might occur because of some uncertainty as to what is target and what is mask (Wolford \& Chambers, 1983), or, perhaps, because some attention is automatically allocated to the masks as well as to the target. We will refer to this as competition for attentional resources. A third component occurs when the masks are drawn from the set of possible targets. Performance is facilitated when the target and mask are assigned to the same response, but inhibited when the target and mask are assigned to different responses (Eriksen \& Eriksen, 1974). We will refer to this as response competition.

Wolford and Chambers (1983) argued that all three factors were involved in lateral masking. Their relative in fluences depend on a variety of factors such as the spacing between the target and the mask, the type of masks used, and the configuration of the display in terms of possibilities for perceptual grouping. They further argued that contour interaction was especially prevalent when the target and the mask were closely spaced, and demonstrated that lateral masks have qualitatively different effects at close spacing than they do at wider spacing. They concluded that, at close spacing, contour interaction was the

We would like to thank Carol Fowler for her helpful comments on the manuscript. Reprint requests should be sent to George Wolford, Department of Psychology, Dartmouth College, Hanover, NH 03755. dominant factor in lateral masking, but that at more distant spacing contour interaction ceased to play a role. La Heij and van der Heijden (1983) also argue that contour interaction ("feature-specific interference," in their terms) is obtained only at close spacing.

Over what separation does contour interaction play a role? To answer this question, it is necessary to use a paradigm in which the influences of response competition and attentional variables are minimized. Probably the most widely cited study addressing this issue was carried out by Flom et al. (1963). They presented subjects with Landolt Cs centered on the fovea. Subjects were given unlimited viewing time. Each $\mathrm{C}$ contained a near-threshold gap in one of four locations and the $\mathrm{Cs}$ were surrounded by four bars. The spacing between the Landolt $\mathrm{C}$ and the bars was varied parametrically from $0^{\circ}$ (touching) to $.42^{\circ}$ (the latter figure varied slightly across subjects). Accuracy in locating the gap was near asymptote when the bars were far from the C. As the bars were moved closer, a point was reached at which performance began to decline precipitously. Performance continued to decline for smaller separations, except that performance increased again at $0^{\circ}$ separation for most subjects.

Flom et al. claimed that the limit of contour interaction was easily identified for each of the subjects. For subjects with normally sighted eyes, the limit ranged from $1.9^{\prime}$ to $3.8^{\prime}$ arc, averaging $2.8^{\prime}\left(0.42^{\circ}\right)$.

As mentioned earlier, we wanted to examine the effect of contour interaction in a paradigm that minimizes the effect of decisional and attentional variables. Information about the extent of contour interaction will help to isolate the other components of lateral masking. We believe that the Flom et al. paradigm did minimize the influence of the other components. Response competition was minimized in the Flom et al. paradigm, since the target was always a Landolt $C$ and the masks were a set of straight lines. In addition, the masks always appeared on all four sides of the target and should not have biased the response toward one of the four gap locations. The role of attentional variables should also be limited. Wolford and Chambers (1983) argued that in many cases a part 
of lateral masking is caused by uncertainty as to what is target and what is mask. In the Flom et al. paradigm, this uncertainty should have been at a minimum because the target was always presented at the same retinal location and the target and masks were quite dissimilar.

Flom et al. and related studies are frequently cited by investigators who are attempting either to study or to avoid contour interaction. Even though the experiments reported by Flom et al. are exemplary in many ways, they may have limits in their applicability to other paradigms. In particular, many of the investigators use peripheral targets in at least some of their conditions, yet the targets in Flom et al. were centered on the fovea. The estimates of the extent of contour interaction $\left(.03^{\circ}-.05^{\circ}\right)$ may not apply to targets located in the periphery. Some examples are cited in the Discussion to show that the presence or absence of spacing effects may depend on target eccentricity as well as on the particular spacings used.

There are numerous suggestions in the literature that contour interaction occurs over wider separations in the periphery than in the fovea. Flom et al. argued that this would be the case based on peripheral acuity data. Jacobs (1979) used a paradigm similar to the one used by Flom et al. He obtained evidence that contour interaction did occur at separations in the periphery that would not have led to contour interaction for foveal targets. The performance measures used by Jacobs, however, do not allow the calculation of the extent of contour interaction in the periphery. Bouma (1970) orthogonally varied target eccentricity and target mask separation in a letteridentification paradigm. He found that lateral masking occurred over wider separations as the target was moved into the periphery. He estimated that lateral masking occurs over a separation approximately equal to one-half of the target eccentricity. Bouma (1970), however, made no attempt to isolate the various components of lateral masking. The masks were chosen from the same set as the targets (letters of the alphabet). It is possible, then, that Bouma's estimates do not apply to contour interaction, per se. Perhaps contour interaction occurs over only part of the range estimated by Bouma, with the other components of lateral masking responsible for the remainder.

Our experiment was a replication of Flom et al. and an extension to other target eccentricities. We chose their paradigm because it seems to minimize the roles of attention and response competition. Flom et al. used unlimited exposure durations with the targcts centered on the fovea. We used three target eccentricities: $0^{\circ}, 2^{\circ}$, and $5^{\circ}$ from the center of the fovea and tachistoscopic presentation.

\section{METHOD}

\section{Subjects}

Four members of the Dartmouth community, including the two authors, served as subjects. All four subjects had normal or corrected-to-normal vision. Two subjects were naive concerning the purpose of the experiment. A fifth subject was discarded due to near-perfect accuracy in all of the foveal conditions.

\section{Stimuli and Apparatus}

The stimuli were constructed from dot matrices and were presented on a video monitor controlled by a Terak 8510 a microcomputer. The target was a square with a gap on one of the four sides. The sides were $.20^{\circ}$ long and the gap was $.04^{\circ}$. On each trial, the target was surrounded by four mask lines. The mask lines were $.20^{\circ}$ long and $.04^{\circ}$ wide. The mask lines were presented at one of 12 target mask separations: $.00^{\circ}$ (touching), $.04^{\circ}, .08^{\circ}, .12^{\circ}$, $.16^{\circ}, .20^{\circ}, .24^{\circ}, .28^{\circ}, .48^{\circ}, .60^{\circ}, .80^{\circ}$, or infinity (no mask lines). The targets were presented at one of three eccentricities: $0^{\circ}, 2^{\circ}$, or $5^{\circ}$ from the center of the fovea. The peripheral targets were presented randomly to the right or left visual field. The viewing distance of $71 \mathrm{~cm}$ was controlled by a wooden hood with an eyepiece attached to the front of the monitor. The target and masks were presented as black lines on a white background.

\section{Procedure}

Each subject completed 10 sessions, usually on separate days. The first session was treated as practice. Each session consisted of six blocks, two blocks at each target eccentricity. Target eccentricity was constant within a block. Each block consisted of 96 trials, the 12 target-mask separations $\times$ the 4 gap locations (up, down, left, or right) $\times$ the 2 visual fields. For foveal blocks, the trials were repeated twice, as visual field did not vary. Within a session, the order of the blocks was randomized, and within a block, the order of the trials was randomized.

The subjects initiated each trial by pressing a key. The trial began with a $1-\sec$ presentation of a fixation cross. The fixation cross was $.2^{\circ}$ high and $.2^{\circ}$ wide. The subjects were instructed to maintain fixation on the cross. The target was then presented for a predetermined duration. The subjects responded by pressing one of four keys to indicate the location of the gap. The keys were arranged in a pattern to correspond to the four possible gap locations.

Because we wanted to compare performance functions at each eccentricity, we felt that it was important to maintain similar levels of absolute performance at each eccentricity. To accomplish this, we varied stimulus duration independently for each subject and for each eccentricity. Our goal was to keep performance at $80 \%$ on the no-mask displays. (Flom et al. varied gap width to achieve the same goal.) Stimulus duration was adjusted at the start of a session on the basis of the results of the preceding session. Because we used a raster scan display, stimulus duration had to be adjusted in multiples of $16.67 \mathrm{msec}$. For foveal displays, stimulus duration varied between 17 and $33 \mathrm{msec}$ (rounded to nearest millisecond). For displays at $2^{\circ}$ eccentricity, stimulus duration varied between 83 and $100 \mathrm{msec}$. For displays at $5^{\circ}$ eccentricity, stimulus duration varied between 133 and $200 \mathrm{msec}$. We were reasonably successful at maintaining the desired performance levels on the nomask displays, except for foveal targets. Some subjects exceeded the $80 \%$ accuracy criterion at $17 \mathrm{msec}$, our shortest possible duration. For most subjects, stimulus duration at a given eccentricity remained constant after the practice session.

The subjects were given feedback on their accuracy at the end of each block. A session lasted approximately $40 \mathrm{~min}$.

\section{RESULTS}

Across the nine sessions, each subject contributed 144 observations per point for each of the 12 target-mask separations at each of the three target eccentricities. The functions for individual subjects at each eccentricity are shown 

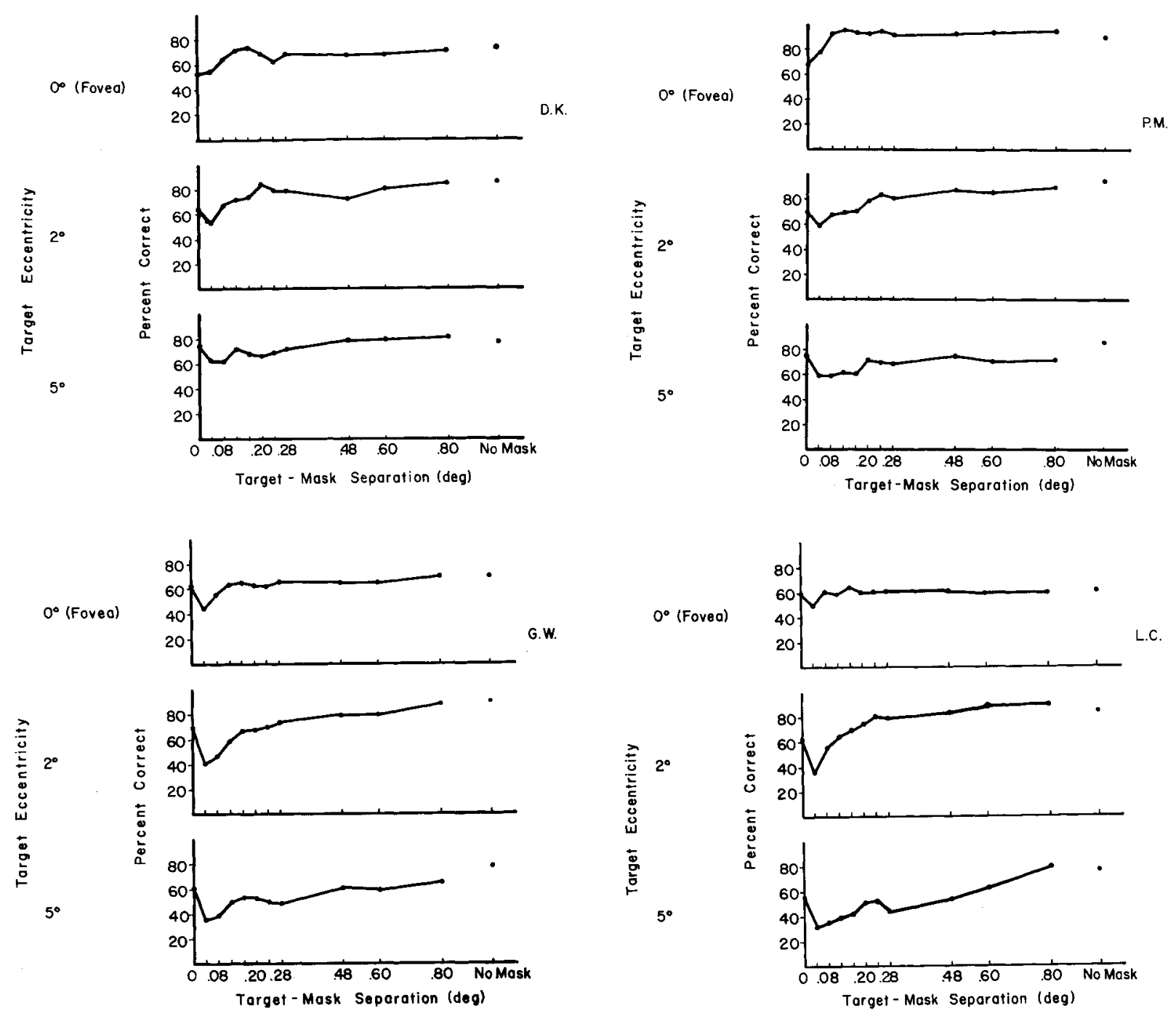

Figure 1. Percent correct target detection by target-mask separation, for each of the four subjects at each retinal eccentricity.

in Figures 1a-1d. In all cases, the data were collapsed over target orientation and visual field. The data were very stable across sessions. The standard errors for single points for individual subjects ranged from a low of $.8 \%$ to a high of $4.2 \%$.

We carried out an analysis of variance on the data. There were two factors: target eccentricity and targetmask separation. Target eccentricity was not significant. This was due, at least in part, to our attempt to equate performance at the different eccentricities by varying exposure duration. The effect of target-mask separation was highly significant $[F(11,33)=23.15, p<.001]$. The interaction of the two factors was also highly significant $[F(22,66)=6.25, p<.001]$. The significant interaction supports the claim that the extent of contour interaction varies with target eccentricity.

As in Flom et al., we attempted to identify the separation at which the masks ceased to have a marked effect on performance. We looked for a point at which performance first reached asymptotic level or a point at which the rate of improvement slowed markedly, a prominent inflection point. When the targets were centered on the fovea, the extent of contour interaction appeared easy to identify. All subjects experienced masking at $.04^{\circ}$. Two subjects (P.M. and L.C. ) reached asymptote at $.08^{\circ}$. The other two subjects (G.W. and D.K.) exhibited some masking at $.08^{\circ}$, but reached asymptote at $.12^{\circ}$. For foveal targets, then, the extent of contour interaction is greater than $.04^{\circ}$ but less than $.12^{\circ}$. This range is consistent with the estimates provided by Flom et al.

When the targets were placed $2^{\circ}$ into the periphery, there was more variability among subjects in the extent of contour interaction. All subjects showed strong masking effects at close spacings. One subject (D.K.) had an inflection point at $.20^{\circ}$; two other subjects (L.C. and P.M.) had inflection points at $.24^{\circ}$. The extent of contour interaction for the final subject (G.W.) is more difficult to identify. The function for that subject rises sharply from $.04^{\circ}$ to $.16^{\circ}$. There is an inflection point at $.16^{\circ}$, but the function continues to rise across the re- 
maining separations. The extent of contour interaction at $2^{\circ}$ eccentricity, then, was between $.20^{\circ}$ and $.24^{\circ}$ for all but one of the subjects.

With targets placed $5^{\circ}$ into the periphery, there was substantial masking at most separations. Two subjects (G.W. and P.M.) did not exhibit any inflection points but continued to improve in a steady fashion across all separations. One subject (L.C.) reached asymptote at $.80^{\circ}$ and the final subject (D.K.) essentially reached asymptote at $.48^{\circ}$. The extent of contour interaction at $5^{\circ}$, then, varies across subjects. Contour interaction at $5^{\circ}$ eccentricity is probably present at half a degree for most subjects and absent by $1^{\circ}$ for most subjects.

\section{DISCUSSION}

Our results show that the extent of contour interaction does vary as a function of the retinal eccentricity of the target. Averaged across subjects, the extent of contour interaction for targets at the center of the fovea was about $.10^{\circ}$. The extent of contour interaction for targets $2^{\circ}$ into the periphery was about $.24^{\circ}$, and the extent for targets $5^{\circ}$ into the periphery was about $.80^{\circ}$.

Our estimates for the extent of contour interaction are much smaller than the estimates provided by Bouma (1970). As mentioned in the introduction, there may not be any contradiction in the differing estimates, since Bouma was attempting to estimate the extent of lateral masking, whereas we were trying to estimate the extent of one component of lateral masking, namely contour interaction.

Investigators who wish to study some aspect of contour interaction (as opposed to lateral masking in general) would probably profit from using target-mask separations that are smaller than the estimates provided above. Those estimates refer only to the maximum extent of contour interaction. In every case, contour interaction was most pronounced at the closest (nontouching) separations. Investigators who wish to avoid contour interaction should use target-mask separations wider than those provided by the estimates above.

Our functions showing the extent of contour interaction at different target eccentricities may help to resolve some apparent inconsistencies in the literature. Some investigators have reported large effects of target-mask spacing, whereas others have failed to find spacing effects. For instance, Estes (1982) presented targets at $1.6^{\circ}$ or $4.8^{\circ}$ and varied target-mask spacing between $.08^{\circ}$ and $.23^{\circ}$. He found a large effect of spacing, as would be predicted from our functions. Kahneman and Henik (1977) presented digits anywhere from the center of the fovea to $1.78^{\circ}$ from the center. They varied spacing from $.52^{\circ}$ to $1.18^{\circ}$ and reported no effect of spacing. Based on our functions, their closest spacing is beyond the range of contour interaction, so no spacing effect would be expected. Santee and Egeth (1982) used separations of $.2^{\circ}$, $.6^{\circ}$, and $1.8^{\circ}$ and reported no effect of spacing. In order to accomplish the varied spacing, however, they presented the stimuli around circles with increasing diameters. Tar- get eccentricity, then, varied with spacing. The target eccentricities were $.12^{\circ}, .36^{\circ}$, and $1.08^{\circ}$. Based on our functions, all of their separations would be outside the range of contour interaction.

Both in Flom et al. and in most of our conditions, performance was better when the masks were touching the targets than it was at the next closest spacing. Several of the subjects claimed that the touching masks altered the appearance of the target rather than destroying or degrading it. With the touching lines, the target often appeared as a fat square with a dot on one side rather than a thinner square with a gap. The location of the dot correlated well with the location of the gap. The use of this information appeared to vary across subjects.

In Wolford and Chambers (1983), we discuss some suggestive findings from the literature on receptive field size. In particular, Hubel and Wiesel (1977) estimated that aggregate receptive fields in monkeys were $.1^{\circ}$ in diameter at the center of the fovea and $.5^{\circ}$ in diameter $7^{\circ}$ into the periphery. These estimates are within the range of our current estimates for contour interaction. An aggregate receptive field is the combined receptive field of all of the cells in a single column of the visual cortex. It is possible that two contours interact if they fall in the same aggregate receptive field.

\section{REFERENCES}

Banks, W. P., Larson, D. W., \& Prinzmetal, W. (1979). Asymmetry of visual interference. Perception \& Psychophysics, , 25, 447-456.

Bouma, H.(1970). Interaction effects in parafoveal letter recognition. Nature, 226, 177-178.

ERIKSEN, B. A., \& ERIKSEN, C. W. (1974). Effects of noise letters upon the identification of a target letter in a nonsearch task. Perception and Psychophysics, 16, 143-149.

EsTES, W. K. (1982). Similarity-related channel interactions in visual processing. Journal of Experimental Psychology: Human Perception and Performance, 8, 353-382.

Estes, W. K. , \& Wolford, G. L.(1971). Effects of spaces on report from tachistoscopically presented letter strings. Psychonomic Science, 25, 77-80.

Flom, M. C., Weymouth, F. W., \& Kahneman, D. (1963). Visual resolution and contour interaction. Journal of the Optical Society of America, 53, 1026-1032.

Hubel, D. H., \& Wiesel, T. N. (1977). Ferrier lecture: Functional architecture of macaque monkey visual cortex. Proceedings of the Royal Society of London (Series B), 198, 1-59.

JACOBS, R. J. (1979). Visual resolution and contour interaction in the fovea and periphery. Vision Research, 19, 1187-1195.

Kahneman, D., \& Henik, A. (1977). Effects of visual grouping on immediate recall and selective attention. In S. Dornic (Ed.), Attention and performance VI. Hillsdale, NJ: Erlbaum.

La Heis, W., \& VAN der heIJden, A. H. C. (1983). Feature-specific interference in letter identification. Acta Psychologica, 53, 37-60.

Mackworth, N. H. (1965). Visual noise causes tunnel vision. Psychonomic Science, 3, 67-68.

Santee, J. L., \& Egeth, H. E. (1982). Independence versus interference in the perceptual processing of letters. Perception \& Psychophysics, 31, 101-116.

Wolford, G., \& Chambers, L. (1983). Lateral masking as a function of spacing. Perception \& Psychophysics, 33, 129-138.

(Manuscript received April 23, 1984; revision accepted for publication September 18, 1984.) 